\title{
A Highly Photostable Hyperbranched Polyglycerol-Based NIR Fluorescence Nanoplatform for Mitochondria-Specific Cell Imaging
}

\author{
Chunhong Dong, Zhongyun Liu, Junqing Liu, Changzhu Wu, Falko Neumann, \\ Hanjie Wang, Monika Schäfer-Korting, Burkhard Kleuser, Jin Chang,* Wenzhong Li, \\ Nan Ma,* and Rainer Haag*
}

Considering the critical role of mitochondria in the life and death of cells, non-invasive long-term tracking of mitochondria has attracted considerable interest. However, a high-performance mitochondria-specific labeling probe with high photostability is still lacking. Herein a highly photostable hyperbranched polyglycerol (hPG)-based near-infrared (NIR) quantum dots (QDs) nanoplatform is reported for mitochondriaspecific cell imaging. Comprising NIR Zn-Cu-In-S/ZnS QDs as extremely photostable fluorescent labels and alkyl chain $\left(\mathrm{C}_{12}\right)$ /triphenylphosphonium (TPP)-functionalized hPG derivatives as protective shell, the tailored QDs@hPG- $\mathrm{C}_{12} / \mathrm{TPP}$ nanoprobe with a hydrodynamic diameter of about $65 \mathrm{~nm}$ exhibits NIR fluorescence, excellent biocompatibility, good stability, and mitochondria-targeted ability. Cell uptake experiments

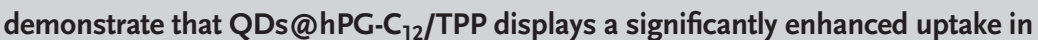
HeLa cells compared to nontargeted QDs@hPG- $\mathrm{C}_{12}$. Further co-localization study indicates that the probe selectively targets mitochondria. Importantly, compared with commercial deep-red mitochondria dyes, QDs@hPG-C 12 /TPP possesses superior photostability under continuous laser irradiation, indicating great potential for longterm mitochondria labeling and tracking. Moreover, drug-loaded QDs@hPG- $\mathrm{C}_{12} / \mathrm{TPP}$ display an enhanced tumor cell killing efficacy compared to nontargeted drugs. This work could open the door to the construction of organelle-targeted multifunctional nanoplatforms for precise diagnosis and high-efficient tumor therapy.

\section{Introduction}

Mitochondria, the powerhouse and suicidal weapon store inside most eukaryotic cells, exert both vital and lethal functions in physiological and pathological scenarios. ${ }^{[1]}$ Mitochondria dysfunctions led to many human diseases. ${ }^{[2]}$ Numerous mutations of mitochondrial DNA contribute to an increased risk of developing some malignancies. ${ }^{[3]}$ Mitochondria in tumor cells are structurally and functionally different from the normal counterparts. ${ }^{[4]}$ Compared with nonimmortalized normal cells, tumor cells are more susceptible to mitochondrial perturbations, which make mitochondria become attractive alternative targets for cancer therapeutics. ${ }^{[5]}$ Mitochondrialtargeted treatment could achieve better anticancer effect and help to overcome multidrug resistance in tumor chemotherapy, representing a promising approach to eradicate chemotherapy-refractory cancer cells. ${ }^{[6]}$ Despite great efforts have been
C. Dong, Dr. J. Liu, Dr. H. Wang, Prof. J. Chang

School of Life Sciences

School of Materials Science and Engineering

Tianjin Engineering Center of Micro-Nano Biomaterials

and Detection-Treatment Technology

Collaborative Innovation Center of Chemical Science and Engineering

Tianjin University

Tianjin 300072, P. R. China

E-mail: jinchang@tju.edu.cn

Dr. Z. Liu

Key Laboratory of Coastal Environmental Processes

and Ecological Remediation

Research Center for Coastal Environmental Engineering

and Technology of Shandong Province

Yantai Institute of Coastal Zone Research

Chinese Academy of Sciences

Yantai, Shandong 264003, P. R. China

Dr. C. Wu, F. Neumann, Prof. N. Ma, Prof. R. Haag

Institut of Chemie and Biochemie

Freie Universität Berlin

Takustr. 3, 14195 Berlin, Germany

E-mail: haag@chemie.fu-berlin.de
Prof. M. Schäfer-Korting

Institut für Pharmazie

Freie Universität Berlin

Königin-Luise-Str. 2-4, 14195 Berlin, Germany

Prof. B. Kleuser

Institut für Ernährungswissenschaft

Universität Potsdam

Arthur-Scheunert-Allee 114-116, 14558 Nuthetal, Germany

Dr. W. Li

Reference and Translation Center for Cardiac Stem Cell Therapy

Universität Rostock

Schillingallee 69, 18057 Rostock, Germany

Prof. N. Ma

Institute of Biomaterial Research

Helmholtz-Zentrum Geesthacht

Kantstraße 55, 14513 Teltow-Seehof, Germany

E-mail:nan.ma@hzg.de

DOI: 10.1002/adhm.201600212 
made on tumor cell-targeted nanoparticle systems for tumor diagnosis and therapy, the development of mitochondria-targeted nanoparticle systems is still in its infancy. Therefore, it is of great importance to fabricate a mitochondria-targeted nanosystem to reach the aim of mitochondria-specific imaging for deeper understanding of tumor mitochondria, advancing early tumor diagnosis and high-performance tumor theranostics.

Up to now, some attempts have been made on developing gold nanoclusters $(\mathrm{Au} \mathrm{NCs}),{ }^{[7]}$ quantum dot $(\mathrm{QD}){ }^{[8]}$ magnetic nanoparticles, ${ }^{[8 a]}$ and carbon nanotube ${ }^{[9]}$ in order to overcome the poor photostability of currently available mitochondrial dyes (such as Rhodamine, MitoTracker, JC1 dye, etc.). Among them, fluorescent nanoparticles have gained particular great interest by virtue of their excellent optical properties and lowcost, convenient, and high sensitive detection. Recently, Liu and co-workers have developed a fluorescent AuNCs@CS-TPP probe with excellent photostability and strong fluorescence emission. ${ }^{[7]}$ Jana's group has constructed a triphenylphosphonium (TPP) functionalized CdSe QDs nanoprobes for mitochondria imaging by optimized surface chemistry. ${ }^{[8 b]}$ These studies represent a "step forward" toward the development of stable mitochondria imaging agents. However, there are still many outstanding problems in terms of fluorescence property, safety, and versatility that required immediate solution, if a translational goal is to be achieved. The bluish or green fluorescence-emitted $\mathrm{Au} \mathrm{NCs}$ and CdSe QDs suffered from interference of biological autofluorescence and limited tissue penetration depth, limiting their application. By contrast, nearinfrared (NIR) fluorescence displays relatively low autofluorescence. Besides, the biocompatibility of the nanoprobes needs to be further optimized by optimizing surface modification and avoiding toxic cadmium or lead-induced toxicity. Moreover, the previously reported nanoprobes were designed only for single imaging performance, lacking versatility and flexibility for further therapeutic applications. So, a biocompatible multifunctional mitochondria-targeted NIR fluorescence nanoplatform remains highly desirable.

Previously, we have reported the synthesis of cadmium-free NIR Zn-Cu-In-S/ZnS (ZCIS/ZnS) QDs which have drawn intense attention in the field of biolabeling and tumor diagnosis owing to their low toxicity and superior optical properties. ${ }^{[10]}$ Their emitted NIR fluorescence could help to reduce biological autofluorescence and background signals, facilitating precise imaging. ${ }^{[11]}$ Herein, we aimed to develop a high-performance mitochondria-specific ZCIS/ZnS QDs nanoplatform via elaborate surface engineering. Polyethylene glycol (PEG) is a gold standard for improved biocompatibility, hydrophilicity, stability, and long-circulation property of nanoparticles by taking advantage of hydrogen bonding and steric stabilization effect of the ether groups. ${ }^{[12]}$ However, the only one or two attachment sites limited their multifunctionalization capability. For the present study, a PEG-analogous polymer, hyperbranched polyglycerol (hPG) which has been identified to be a promising biocompatible alternative to PEG, was used to engineer ZCIS/ZnS QDs for the first time. As a highly biocompatible branched aliphatic polyether, hPG possesses not only physical properties analogous to PEG, favorable low toxicity, and low nonspecific adsorption of serum albumin but also multiple attachment sites which facilitate the attachment of other functionalities to construct flexible macromolecular assembled nanostructures. ${ }^{[13]}$ It has been widely used for constructing biocompatible nanogels, biomimetic materials for targeted drug delivery, and surface antifouling. Moreover, dendritic amphiphiles with highly branched structures have been reported to be more stable micellar aggregates than linear amphiphiles. ${ }^{[14]}$ All these make it an ideal candidate for the construction of versatile and multifunctional QDs nanoplatform.

In the present study, we developed an hPG-based QDs nanoplatform by incorporating near-infrared ZCIS/ZnS QDs into the assembly of our tailored hPG derivatives, amphiphilic hPG-alkyl chain (hPG-C 12 ), and hPG-TPP. The performance of the QDs@ hPG-C ${ }_{12} /$ TPP nanoprobe for mitochondria-targeted imaging is illustrated in Figure 1. The introduction of TPP, an amphiphilic cation with a delocalized positive charge, could facilitate cellular uptake, effective escape from endolysosomes, and further transportation process of crossing highly dense and impermeable mitochondrial membrane. ${ }^{[15]}$ And ZCIS/ZnS QDs could serve as photostable near-infrared fluorescent labels, facilitating long-term tracking of mitochondria with little biological autofluorescence and reduced background signals. The obtained QDs nanoprobe demonstrated low cytotoxicity, mitochondria-targeting ability, and superior photostability under continuous laser irradiation. Moreover, mitochondria-targeted drug-loaded QDs@ hPG-C Cl2 $_{12}$ TPP displayed enhanced tumor cell killing efficacy than nontargeted ones, indicating the great potential of QDs nanoplatform for mitochondria-acted enhanced tumor therapy. To the best of our knowledge, this is the first time to construct a mitochondria-specific NIR QDs nanoplatform with PEG-analogous hyperbranched polyglycerol. This work could open a new door to the construction of organelle-targeted multifunctional nanoplatforms for precise diagnosis and high-efficient tumor therapy.

\section{Results and Discussion}

\subsection{Synthesis and Characterization of the hPG Derivatives}

Given that high-quality ZCIS/ZnS QDs with hydrophobic 1-dodecanethiol (DDT) ligands cannot be directly used in physiological environment, hPG-based amphiphiles were hence synthesized to encapsulate the hydrophobic QDs by hydrophobic interactions. Besides, to achieve the specificity to intracellular mitochondria, a mitochondriotropic amphiphilic cation TPP that possessed a delocalized positive charge was introduced to facilitate selective accumulation of nanoparticles in mitochondria. The delocalized positive charge combines good biocompatibility with efficient membrane fusion, cellular uptake, effective escape from endosomes and lysosomes after cellular uptake, and ultimate mitochondrial targeting. ${ }^{[15]}$ In order to meet the requirements for effective QDs encapsulation and mitochondria targeting, hPG derivatives including hPG- $\mathrm{C}_{12}$ and hPG-TPP were tailored and synthesized (Figure 2A). Molecular structure and GPC result of hPG we used are shown in Figure S1 (Supporting Information). Amphiphilic $\mathrm{hPG}-\mathrm{C}_{12}$ was readily synthesized by the esterification reaction between terminal hydroxyl groups of hPG and lauroyl chloride. The attached long carbon chains $\left(\mathrm{C}_{12}\right)$ of lauric chloride (LC) acted as the hydrophobic segment of the hPG-based amphiphiles, providing the requisite amphiphilic property. Given the mitochondria targeting ability, 


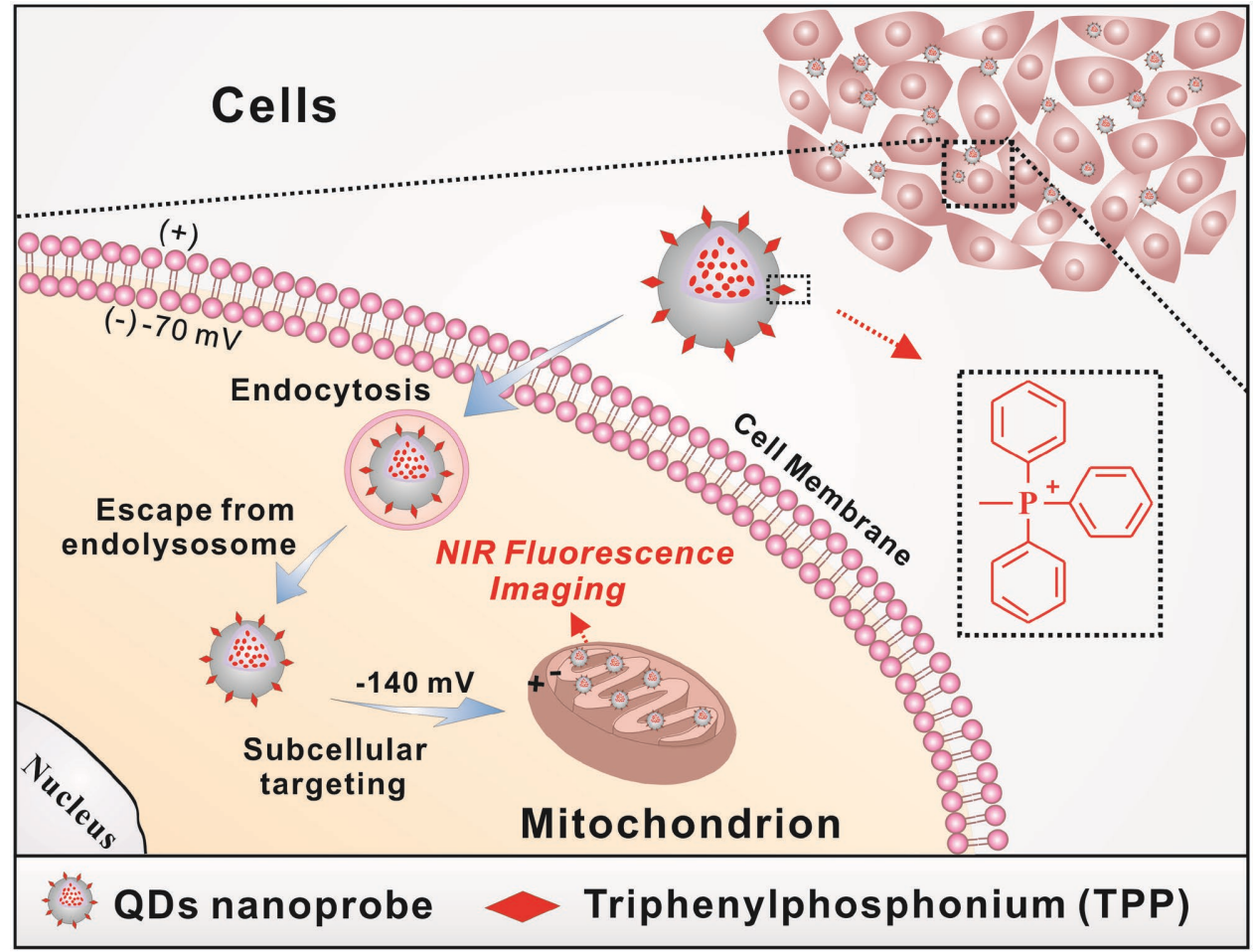

Figure 1. Schematic illustration of hyperbranched polyglycerol-based QDs@hPG-C $\mathrm{C}_{12} / \mathrm{TPP}$ for mitochondria-specific cell imaging.

triphenylphosphonium moieties were introduced. Owing to the low esterification reaction activity between the terminal hydroxyl of hPG and the carboxyl of (4-carboxybutyl) triphenylphosphonium bromide (CTPP) in our former trials, amine functionalized hPG (hPG- $\mathrm{NH}_{2}$ ) was first prepared here by a three-step protocol. The intermediates and final hPG- $\mathrm{NH}_{2}$ were characterized by Fourier transform infrared (FTIR) spectroscopy (Figure S2, Supporting Information). Then, hPG-TPP was prepared by conjugating CTPP to $\mathrm{hPG}-\mathrm{NH}_{2}$ on the basis of carbodiimide chemistry with 1-ethyl-3-(3-dimethylaminopropyl) carbodiimide hydrochloride (EDC. $\mathrm{HCl}$ ) and $N$-hydroxysuccinimide (NHS) as the effective cross-linker. Corresponding ${ }^{1} \mathrm{H}$ NMR and FTIR spectra of the hPG derivatives are demonstrated in Figure $2 \mathrm{~B}-\mathrm{E}$. Compared to the ${ }^{1} \mathrm{H}$ NMR spectrum of pure hPG in Figure $2 \mathrm{~B}$, the additional signals peaks at (a) $0.90 \mathrm{ppm}$, (b) at $1.30 \mathrm{ppm}$, (c) at $1.62 \mathrm{ppm}$, and (d) at $2.36 \mathrm{ppm}$ in Figure $2 \mathrm{C}$ were attributed to $\mathrm{H}$ protons of lauroyl chloride, demonstrating the successful introduction of hydrophobic $\mathrm{C}_{12}$. The grafting percentage of $C_{12}$ to hydroxyl groups of $h P G$ was about $11 \%$, as calculated by dividing the integration of the peak areas at $0.90 \mathrm{ppm}$ by that at $3.45-3.90 \mathrm{ppm}$. Meanwhile, the distinctive signal peak at 7.78-7.89 ppm derived from benzene rings suggested the success of (4-carboxybutyl) triphenylphosphonium conjugation (Figure 2D). The calculated grafting percentage of TPP to hydroxyl groups of hPG was about $5 \%$. The FTIR spectra in Figure 2E further confirmed this by the appearance of respective characteristic absorption peak of ester bond at $1725 \mathrm{~cm}^{-1}$ and absorption peak of benzene from 1600 to $1400 \mathrm{~cm}^{-1}$.

In general, amphiphilic polymers can spontaneously selfassemble into spherical nanostructures in water. The amphiphilic hPG-C ${ }_{12}$ bears hydrophilic polyglycerol and hydrophobic alkyl chains. The self-assembly and aggregation behavior triggered by hydrophobic interactions among the peripheral hydrophobic segment of alkyl chains was studied by measuring critical aggregation concentration (CAC) in deionized water with hydrophobic pyrene as a model fluorescence probe. From the fluorescence emission spectra of pyrene at various concentrations of hPG$\mathrm{C}_{12}$ from 0 to $0.5 \mathrm{mg} \mathrm{mL}^{-1}$, we could easily observe the intensity change of various peaks as the concentration increased (Figure S3, Supporting Information). The change of fluorescent intensity ratio $\left(I_{373} / I_{384}\right)$ was plotted against the logarithm concentrations of the $\mathrm{hPG}-\mathrm{C}_{12}$. The CAC value of the $\mathrm{hPG}-\mathrm{C}_{12}$ calculated by the interception of two straight lines was about $0.83 \mathrm{mg} \mathrm{L}^{-1}$. The quite low $\mathrm{CAC}$ value indicated that $\mathrm{hPG}-\mathrm{C}_{12}$ assemble very easily into micelle in water, reflecting its great efficiency and potential for encapsulating hydrophobic components, including small molecules and nanoparticles.

\subsection{Fabrication and Characterization of the Mitochondria- Targeted QDs@hPG-C 12 /TPP QDs Nanoprobe}

In this work, high-performance hydrophobic NIR-emitted ZCIS/ZnS QDs were first prepared by using a high temperature organic solution method. Figure S4 (Supporting Information) shows a photograph of just obtained ZCIS/ZnS QDs stock solution. Different batches of QDs with tunable emission peak from 600 to $700 \mathrm{~nm}$ were obtained by varying the doping ratio of $\mathrm{Zn}$ and reaction temperature (S5). Herein, a batch of QDs $\left(\lambda_{\text {em }}=645 \mathrm{~nm}\right)$ was used. The corresponding energy dispersive spectroscopy spectrum was given in Figure S6 (Supporting Information). A simple, fast, and facile ultrasonication-induced 
A

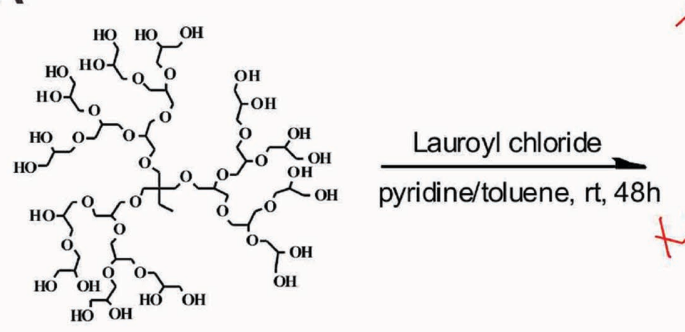

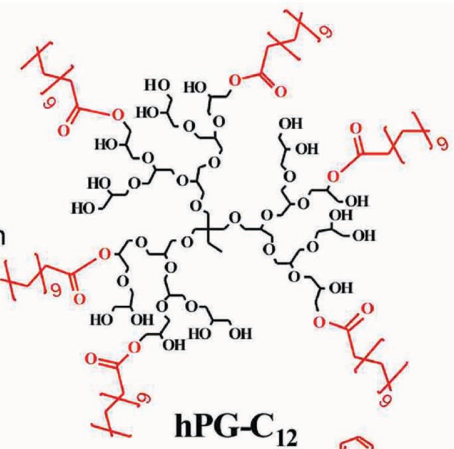

hPG-OH

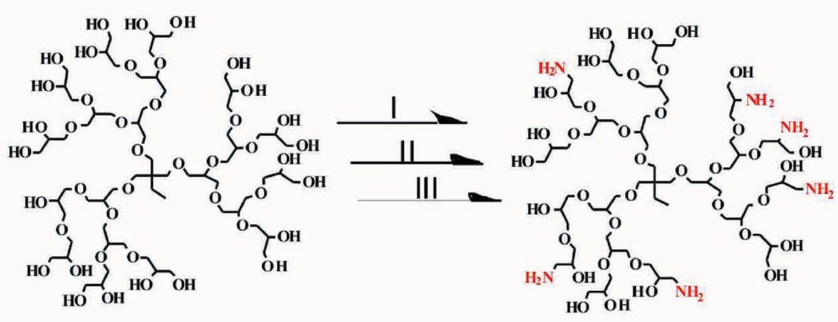

PG-C 12

hPG-OH

hPG-NH

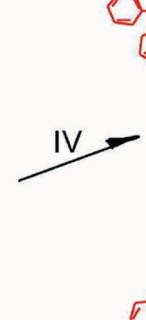<smiles>C1CCCC2(CC1)CCCCC2</smiles>

\section{Synthetic route of hyperbranched polyglycerol derivatives}

B
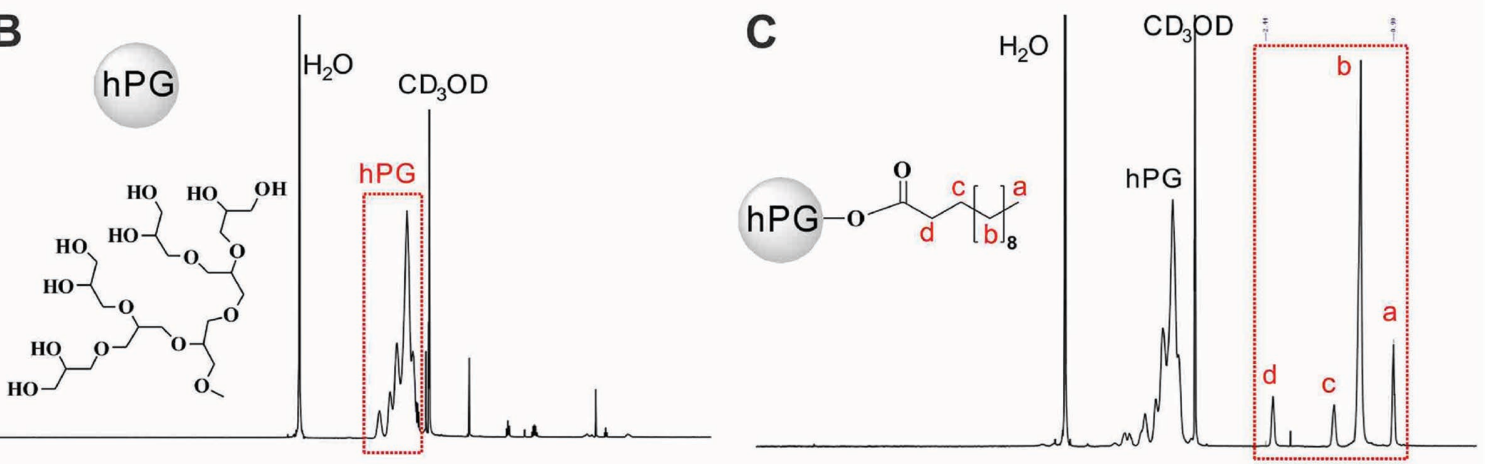

\begin{tabular}{llllllllllllllllllll}
\hline 8.5 & 8.0 & 7.5 & 7.0 & 6.5 & 6.0 & 5.5 & 5.0 & 4.5 & 4.0 & 3.5 & 3.0 & 2.5 & 2.0 & 1.5 & 1.0 & 0.5 & 0.0
\end{tabular}

D

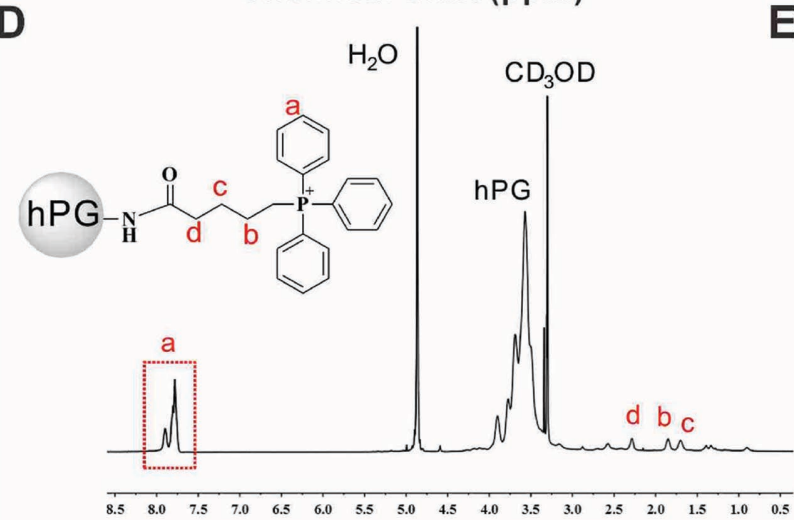

Chemical Shift (ppm)

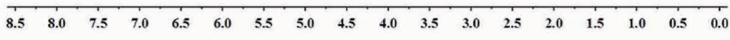

E

Chemical Shift (ppm)

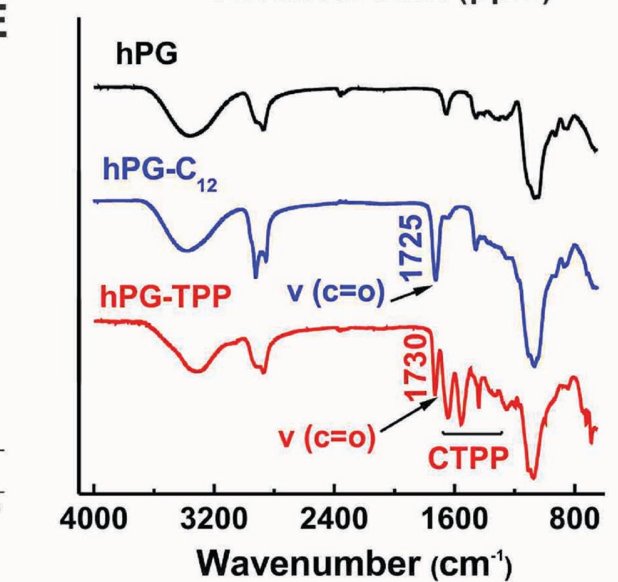

Figure 2. Synthesis and characterization of $h P G$ derivatives. A) Synthetic route of hyperbranched polyglycerol (hPG) derivatives, amphiphilic $h P G-C_{12}$, and TPP-functionalized hPG-TPP. (I) $\mathrm{MsCl}$, pyridine, $\mathrm{N}_{2}, 0^{\circ} \mathrm{C}-\mathrm{rt}, 24 \mathrm{~h}$; (II) $\mathrm{NaN}_{3}$, DMF, $100{ }^{\circ} \mathrm{C}, 12 \mathrm{~h}$; (III) P(Ph) $3, \mathrm{THF} / \mathrm{H}_{2} \mathrm{O}, 45^{\circ} \mathrm{C}, 24 \mathrm{~h}$; (IV) $\mathrm{CTPP}$, EDC/NHS, rt, 48 h. ${ }^{~} \mathrm{H}$ NMR spectra of $B$ ) hPG, C) hPG-C 12 , and D) hPG-TPP. E) FTIR spectra of hPG, hPG-C 12 , and hPG-TPP. 
A

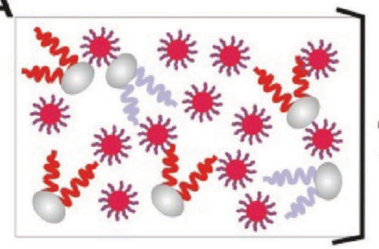

Hydrophobic QD

hPG-C12

C

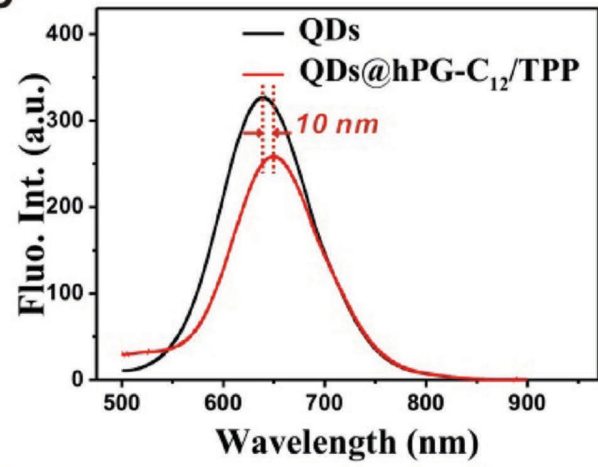

$\mathbf{F}$

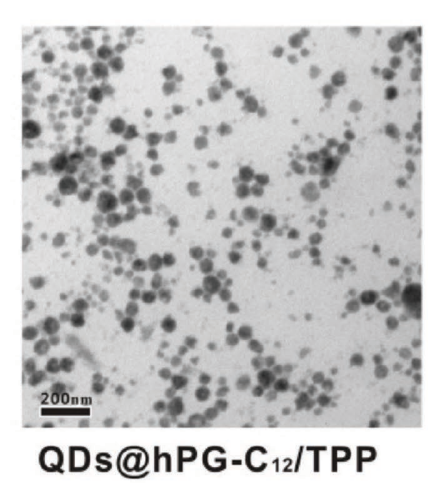

G

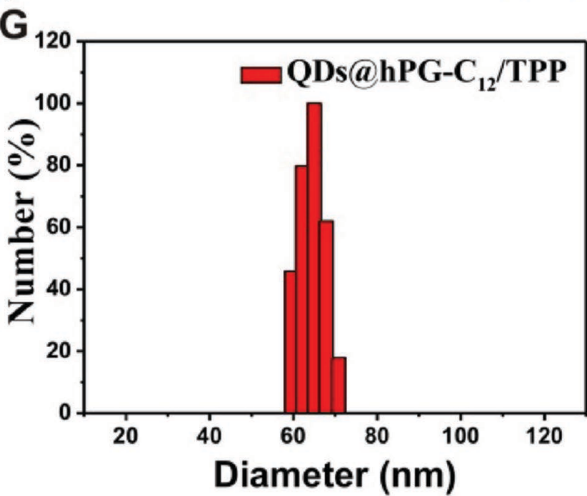

B

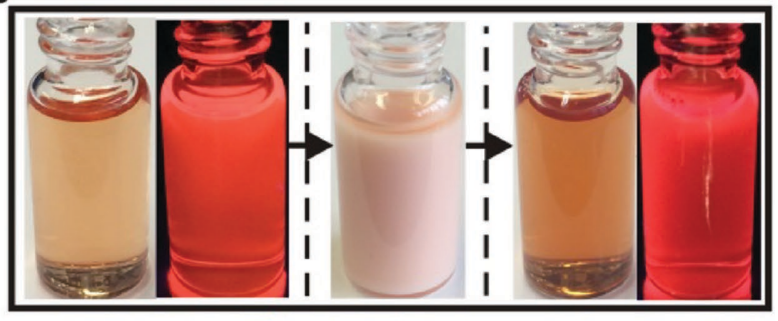

$B_{1}$, chloroform B2, biphase

B3, water

E

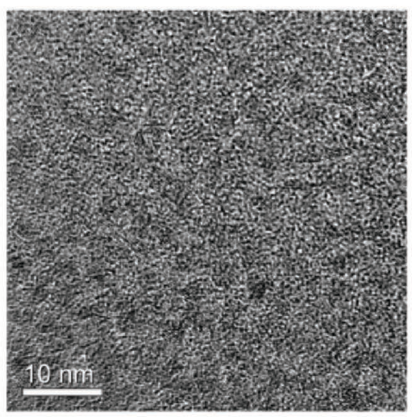

Figure 3. Preparation and characterization of the QDs nanoprobe. A) Schematic illustration of the modification procedure by an ultrasonication-based assembly method. B) The bright-field and dark-field photographs during the process of polymer modification: Hydrophobic ZCIS/ZnS QDs in chloroform $\left(B_{1}\right)$, QDs emulsion system in biphase $\left(B_{2}\right)$, and hydrophilic QDs@hPG- $C_{12} / T P P$ nanoprobes in water $\left(B_{3}\right)$. C) Fluorescence spectra of pure QDs and QDs@hPG-C 12 /TPP. D) UV-vis absorbance spectra of hPG-C 12 , QDs, and QDs@hPG-C 12 /TPP. E,F) TEM images of pure QDs and QDs@ hPG-C $12 / T P P . G)$ DLS result of QDs@hPG-C $12 / T P P . H)$ Zeta potential of QDs@hPG-C $12 / T P P$ nanoprobes.

assembly method was employed to fabricate the QDs nanoprobes (Figure 3A). Upon sonication, the amphiphilic hPG derivatives assembled into micelle-like uniform spherical nanostructures which, in the presence of the hydrophobic ZCIS/ ZS QDs QDs and drug molecules, spontaneously encapsulated them into the inner hydrophobic core of the micelles through strong hydrophobic interactions.

To optimize the assembly condition, we explored the influence of $m_{(\mathrm{hPG}-\mathrm{C} 12)} / m_{(\mathrm{QDs})}$ ratios on the fluorescence property and hydrodynamic size of assembled QDs@hPG-C 12 nanoprobe (Figures S7 and S8, Supporting Information). With the increase of polymer dosage in the measured range, the fluorescence intensity of obtained QDs@hPG-C 12 nanoprobe increased while redshift distance of fluorescence emission spectrum decreased. In the meantime, Z-average size of QDs@hPG- $\mathrm{C}_{12}$ decreased. Taking into consideration of fluorescence property and particle size, an appropriate $m_{\text {(hPG-C12) }} / m_{(\mathrm{QDs})}$ ratio of 7 was employed for our later work. Characterizations of the QDs@hPG-C 12 nanoprobes are shown in Figure S9 (Supporting Information).

To construct the mitochondria-targeted QDs nanoprobe, hPG-TPP was further added with a mass fraction of about $20 \%$. To give a scientific and comprehensive description, visual photographs, fluorescence emission, UV-vis absorption, transmission electron microscopy (TEM) image, hydrodynamic size, zeta potential, and FTIR spectrum of the mitochondria-targeted QDs@hPG-C 12 TPP nanoprobe were characterized and are shown in Figure 3 and Figure S10 (Supporting Information). We could see from the photographs in Figure 3B that a homogeneous emulsion was formed under ultrasonication in the biphase system. After the oil evaporation, the QDs solution became clear and transparent, which indicated the good hydrophilicity of the obtained QDs nanoprobes. As observed 
from dark-field photographs, no obvious fluorescence change occurred after the polymer modification, indicating that most of the QDs fluorescence was well retained. To further qualitatively evaluate the influence of modification process on fluorescence intensity of QDs, fluorescence spectra of pure QDs and obtained QDs nanoprobes were measured at the same QDs concentration (Figure 3C). After modification, QDs@hPG$\mathrm{C}_{12} / \mathrm{TPP}$ nanoprobes reserved about $80 \%$ of original intensity. The well-preserved fluorescence indicated the polymer layer of hPG-based derivative provided complete protection and surface passivation for the hydrophobic QDs. Compared with the UV-vis absorption spectrum of QDs@hPG-C 12 , the appearance of characteristic absorption peak of triphenylphosphonium at $267 \mathrm{~nm}$ in the spectrum of QDs@hPG-C ${ }_{12} /$ TPP indicated successful incorporation of hPG-TPP (Figure 3D and Figure S9B, Supporting Information). As shown in Figure 3E, the original pure ZCIS/ZnS QDs were nearly monodisperse nanoparticles of $\approx 3 \mathrm{~nm}$ in size. After modification, our obtained QDs@ hPG-C $\mathrm{C}_{12} / \mathrm{TPP}$ nanoprobes were well-dispersed uniform nanospheres with a size of 60-70 nm, and no aggregation occurred (Figure 3F). The hydrodynamic size of QDs@hPG-C 12 /TPP nanoprobe exhibited in Figure 3G was about $65 \mathrm{~nm}$ which was in accordance with the size shown in TEM image. The size of QDs@hPG-C ${ }_{12}$ nanoprobe was slightly smaller than nontargeted ones (Figure S9C, Supporting Information). As for the zeta potential, the mitochondria-targeted QDs@hPG-C $12 / T P P$ nanoprobe had a little higher zeta potential $(\approx 7.94 \mathrm{mV})$ than the nontargeted QDs@hPG-C ${ }_{12}$ nanoprobe $(\approx 3.99 \mathrm{mV})$, which was mostly induced by the residual amino groups in hPG and attached triphenylphosphonium groups (Figure $3 \mathrm{H}$ and Figure S9D, Supporting Information). Besides, FTIR spectrum of QDs@hPG-C $\mathrm{C}_{12} / \mathrm{TPP}$ nanoprobe further verified successful incorporation of hPG-TPP by the appearance of characteristic absorption peaks of benzene rings at 1600 and $1580 \mathrm{~cm}^{-1}$ (Figure S10, Supporting Information). All these results demonstrated that hPG derivatives-based QDs@hPG-C 12 /TPP nanoprobe with uniform size and favorable NIR fluorescence emission was successfully fabricated by the ultrasonication-induced assembly method. It was expected that the outer polyglycerol protection layer could provide the nanoprobes with good biocompatibility, excellent stability, reduced nonspecific cellular binding, and mitochondria-specific targeting ability.

\subsection{Size and Fluorescence Stability of the QDs@hPG-C $12 / T P P$ Nanoprobe}

The size and fluorescence stability of QDs nanoprobes is an essential issue for their further applications. In previous report, PEG chains are normally used to conjugate to polymers or onto the surfaces of particles to improve the cell survival and reduce nonspecific protein absorption of nanoparticles. For the present study, as a PEG-analogous polymer, hyperbranched polyglycerol was used to fabricate the mitochondria-targeted QDs nanoprobe. We assumed that similar structures in the repeating unit $-\left(\mathrm{OCH}_{2} \mathrm{CH}\left(\mathrm{CH}_{2} \mathrm{OH}\right)\right) n$ - of polyglycerol could have the same effect and offer excellent stability for the nanoprobes.

To examine the size stability of the QDs@hPG-C 12 /TPP nanoprobe, the prepared QDs nanoprobe was gently dispersed in ultrapure $\mathrm{H}_{2} \mathrm{O}$, PBS buffer (0.1 $\mathrm{m}, \mathrm{pH}$ 7.4), and PBS buffer supplemented with $10 \%$ fetal calf serum (FCS; PBS, 10\% FCS). The particle Z-average size and polydispersity indexes (PDI) were measured by dynamic light scattering (DLS) at different incubation time using a Malvern Zetasizer. As shown in Figure 4A,B, the hydrodynamic size of the QDs@hPG-C $12 / T P P$ nanoprobe in three kinds of solution changed little and the corresponding PDI was almost constant during $10 \mathrm{~d}$ of incubation. Overall, the fabricated QDs@hPG-C $\mathrm{C}_{12} / \mathrm{TPP}$ nanoprobe with hPG-based amphiphiles exhibited excellent size stability. This stability was probably provided by the supermolecular assembly structures of highly branched hPG-based derivatives. ${ }^{[14 a]}$

As for a fluorescence nanoprobe, fluorescence stability in biological fluids is of vital importance for its use in accurate quantitative imaging analysis and in tumor diagnostics. In general, the fluorescence could be easily quenched if the surface coating is not complete and compact enough to completely isolate the hydrophobic QDs from water environment. Herein, fluorescence intensities of the QDs@hPG-C $12 /$ TPP nanoprobes in PBS with $10 \% \mathrm{FCS}$ at $37^{\circ} \mathrm{C}$ were investigated. As demonstrated in Figure 4C, the nanoprobes kept good fluorescence in the measured $10 \mathrm{~d}$ and showed no obvious change in fluorescence intensity, indicating excellent fluorescence stability.

\subsection{In Vitro Cytotoxicity}

The cytotoxicity of the amphiphilic hPG-C 12 , QDs@hPG$\mathrm{C}_{12}$, and QDs@hPG-C ${ }_{12} / \mathrm{TPP}$ nanoprobes against HeLa cells was assessed by using 3-(4,5-dimethylthiazol-2-yl)-2,5diphenyl tetrazolium bromide (MTT) assay. The viability of cells exposed to different concentrations $(12.5,25,50,100$, 200 , and $400 \mu \mathrm{g} \mathrm{mL}^{-1}$ ) of three samples for $24 \mathrm{~h}$ is shown in Figure 4D. With an increase in concentration of QDs@hPG-C 12 and QDs@hPG-C $12 /$ TPP nanoprobes from 25 to $400 \mu \mathrm{g} \mathrm{mL} \mathrm{m}^{-1}$, the cell survival decreased slightly. At the concentration of $400 \mu \mathrm{g} \mathrm{mL} \mathrm{m}^{-1}$, the cell survivals of HeLa cells incubated with the three samples were all above $80 \%$. The hPG-based amphiphilic hPG-C ${ }_{12}$, QDs@hPG-C ${ }_{12}$ nanoprobes, and the mitochondria-targeted QDs@hPG-C $12 /$ TPP nanoprobes all had a low cytotoxicity in the measured concentration range. Although QDs@hPG-C 12 /TPP nanoprobe possessed a slightly higher zeta potential value than QDs@hPG-C C $_{12}$ nanoprobe, we did not observe more toxic effects of this formulation. In particular, the viability of cells incubated with $\mathrm{hPG}-\mathrm{C}_{12}$ was always above $100 \%$. The hPG-based amphiphilic polymer $\mathrm{hPG}-\mathrm{C}_{12}$ did not cause any cytotoxicity against HeLa cells under the concentration of $400 \mathrm{mg} \mathrm{mL}^{-1}$, suggesting low in vitro cytotoxicity. As a biocompatible branched aliphatic polyether analogous to those of PEG, hPG-based amphiphilic hPG- $\mathrm{C}_{12}$ had great potential in the field of constructing biocompatible nanoplatforms via modifying various hydrophobic nanoparticles.

\subsection{Cell Imaging and Mitochondria-Targeted Subcellular Imaging of the QDs@hPG-C $12 /$ TPP Nanoprobe}

To investigate the cell uptake and fluorescence imaging performance of the mitochondria-targeted QDs@hPG-C 12 /TPP 
A
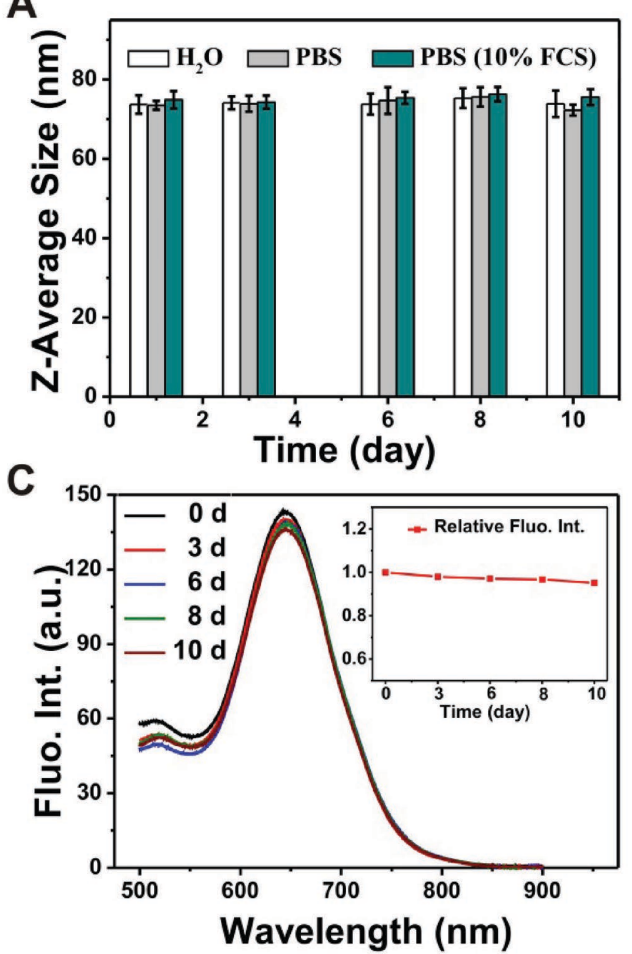

B
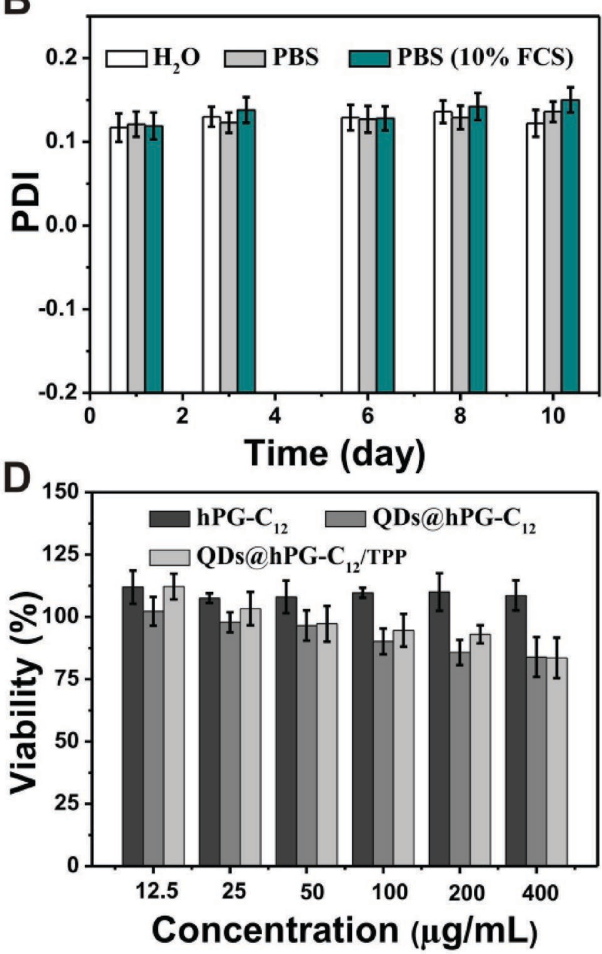

Figure 4. Stability and cytotoxicity of the QDs nanoprobes. A,B) Z-average size and polydispersity index (PDI) stability of QDs@hPG-C $12 / T P P$ in doubly distilled water, PBS buffer ( $\mathrm{pH} 7.4)$, and PBS buffer $(\mathrm{pH} 7.4)$ supplemented with 10\% FCS. C) Fluorescence stability of QDs@hPG-C $12 / T P P$ in PBS buffer ( $\mathrm{pH}$ 7.4) supplemented with 10\% FCS. D) In vitro cytotoxicity studies of hPG-C ${ }_{12}$, QDs@hPG-C 12 , and QDs@hPG-C $12 / T P P$ using the MTT assay in HeLa cells.

nanoprobes, HeLa cells were used as a model and nontargeted QDs@hPG-C 12 /TPP nanoprobes as a control. To test the temporal evolutions, HeLa cells were exposed to QDs nanoprobes for different incubation time periods $(0.5,1.5$, and $3 \mathrm{~h})$. As shown in Figure 5A and Figure S11 (Supporting Information), the fluorescence intensity of QDs in HeLa cells increased with time, which indicated an ongoing cell uptake. By contrast, little nontargeted QDs@hPG-C 12 nanoprobes were internalized by HeLa cells after incubation for $3 \mathrm{~h}$ on account of the extremely low nonspecific absorption of the hyperbranched polyglycerol-based surface coating. The mitochondria-targeted QDs@hPG-C 12 /TPP nanoprobes demonstrated a significantly enhanced cell uptake than nontargeted ones. It was mentioned in previous report that delocalized positive charge in TPP could promote membrane fusion and cellular uptake. ${ }^{[16]}$ Therefore, the introduced triphenylphosphonium moieties effectively promoted the cell uptake of the nanoprobes as a lipophilic cation along with the residual amino groups on hPG. Taken together, the hPG-based polymer coating endowed the nanoprobes excellent biocompatibility and low cytotoxicity, while the introduction of TPP moieties significantly promoted the cell uptake of the QDs nanoprobes. Meanwhile, the flow cytometry analysis further verified the enhanced cell uptake of TPP-decorated QDs@hPG-C 12 /TPP nanoprobe than nontargeted ones (Figure $5 \mathrm{~B}$ ). The change of mean fluorescence intensity of $\mathrm{M}$ area over time is demonstrated in Figure 5C. As time prolonged, the mean fluorescence intensity of M2 area increased significantly from 37.97 to 131.77 in the group of QDs@hPG-C 12 /TPP nanoprobe, while it remained consistently at a low value $(<36)$ in the group of QDs@hPG-C 12 nanoprobe.

To test the feasibility of QDs@hPG-C 12 /TPP nanoprobes for mitochondria-specific imaging, confocal fluorescence images of HeLa cells treated with QDs@hPG-C 12 /TPP were taken by Leica TCS SP5 Confocal Laser Scanning Microscope (CLSM). To avoid mutual fluorescence interference of QDs and mitochondrial staining dye, MitoTracker with excitation wavelength of $644 \mathrm{~nm}$ from Invitrogen was employed for the co-localization study. To distinguish colors of QDs and MitoTracker, Mito Tracker and QDs were set as red and green, respectively. Figure 6A shows confocal images of untreated HeLa cells stained with only Mito Tracker as negative control. No fluorescence in the QDs emission channel was observed in untreated HeLa cells, which suggested that excitation light of $633 \mathrm{~nm}$ had no interference with QDs channel. Further, confocal images of HeLa cells treated separately with nontargeted QDs@hPG-C 12 and targeted QDs@hPG-C 12 /TPP are shown in Figure 6B,C. As shown in Figure 6B, there was almost no QDs fluorescence in the HeLa cells after incubation with nontargeted QDs@hPG-C ${ }_{12}$ for $1.5 \mathrm{~h}$. This was caused by the weak endocytosis of QDs@hPG-C 12 proved in cell uptake experiment. The outer polyglycerol makes it had a quite low cell adsorption and weak uptake. It was previously reported that hPG could reduce nonspecific adsorption to 


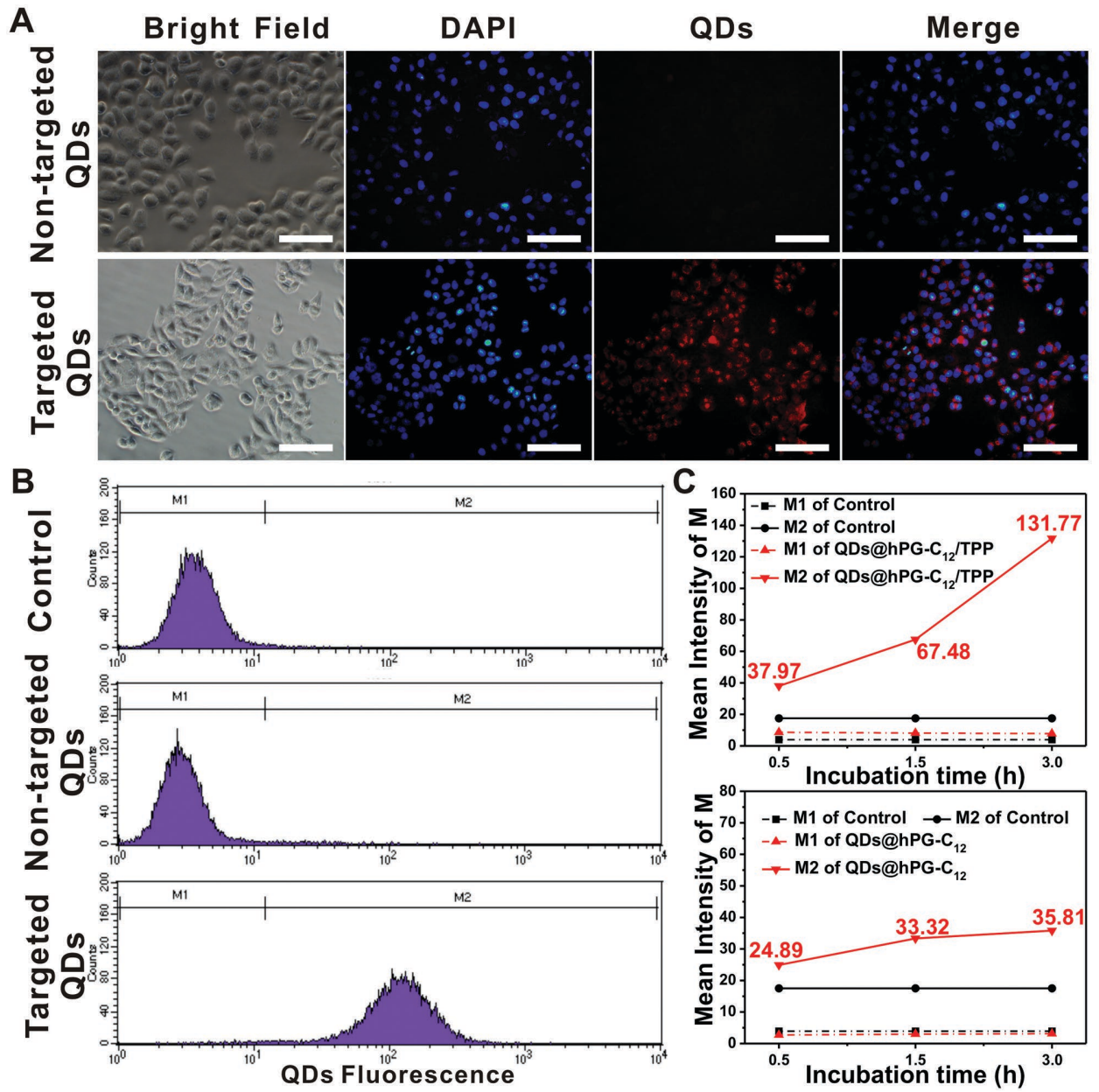

Figure 5. Cellular imaging of the QDs nanoprobes. A) Fluorescent images and B) flow cytometry analysis of HeLa cells incubated separately with nontargeted QDs@hPG-C 12 and mitochondria-targeted QDs@hPG-C $12 / T P P$ nanoprobes at $37^{\circ} \mathrm{C}$ for $3 \mathrm{~h}$. Scale bar represents $\left.100 \mu \mathrm{m} . \mathrm{C}\right) \mathrm{The}$ change of mean fluorescence intensity of $\mathrm{M}$ area in (B) over incubation time. The HeLa cells without any treatment were used as control.

nanoparticles by blocking protein-binding sites and creating a thermodynamic barrier to protein diffusion. By contrast, QDs@hPG-C 12 TPP could be effectively endocytosed into HeLa cells under the same condition and then selectively and specifically accumulate into intracellular mitochondria (Figure 6C). The selectivity was originated from the specific conjugation of TPP with mitochondria, which had been reported in previous work. ${ }^{[18]}$ As a lipophilic cation with three phenyl groups and delocalized positive charge, triphenylphosphonium could ensure effective escape from endosomes and lysosomes facilitate further transportation process of crossing the impermeable mitochondrial membrane. Based on the above analysis, mitochondria-targeted QDs@hPG-C 12 TPP nanoprobe was successfully fabricated. What's more, the nearinfrared fluorescence emission could effectively avoid interference of biological autofluorescence and reduce background signals. In general, our tailor-designed ZCIS/ZnS QDs nanoprobe with near infrared fluorescence emission is an ideal fluorescent nanoparticle for mitochondria-specific labeling, imaging, and tracking in tumor cells.

\subsection{Photostability of QDs@hPG-C 12 /TPP Nanoprobe}

Photostability is very critical for the development of fluorescent probes and their applications for long-term labeling and tracking. To investigate the photostability of our constructed QDs@hPG-C 12 /TPP nanoprobe under irradiation, HeLa cells treated with QDs@hPG-C 12 /TPP and MitoTracker were irradiated by laser confocal microscope with excitation at $453 \mathrm{~nm}$ (50\% laser power) and $633 \mathrm{~nm}$ (15\% laser power), respectively. As shown in Figure 7B, there was almost no change in the fluorescence intensity of QDs@hPG-C 12 /TPP nanoprobes in HeLa cells after $8 \mathrm{~min}$ of irradiation. However, the fluorescence intensity of the MitoTracker decreased gradually as irradiation time prolonged, indicating that the commercially available MitoTracker suffered from poor photostability (Figure 7A). Moreover, the mean fluorescence intensities of selected regions of interest (ROI) at different scanning time were analyzed by CLSM and are shown in Figure 7B. It was easily observed that the fluorescence of MitoTracker gradually bleached under irradiation while that of QDs nanoprobe remained unchanged, 


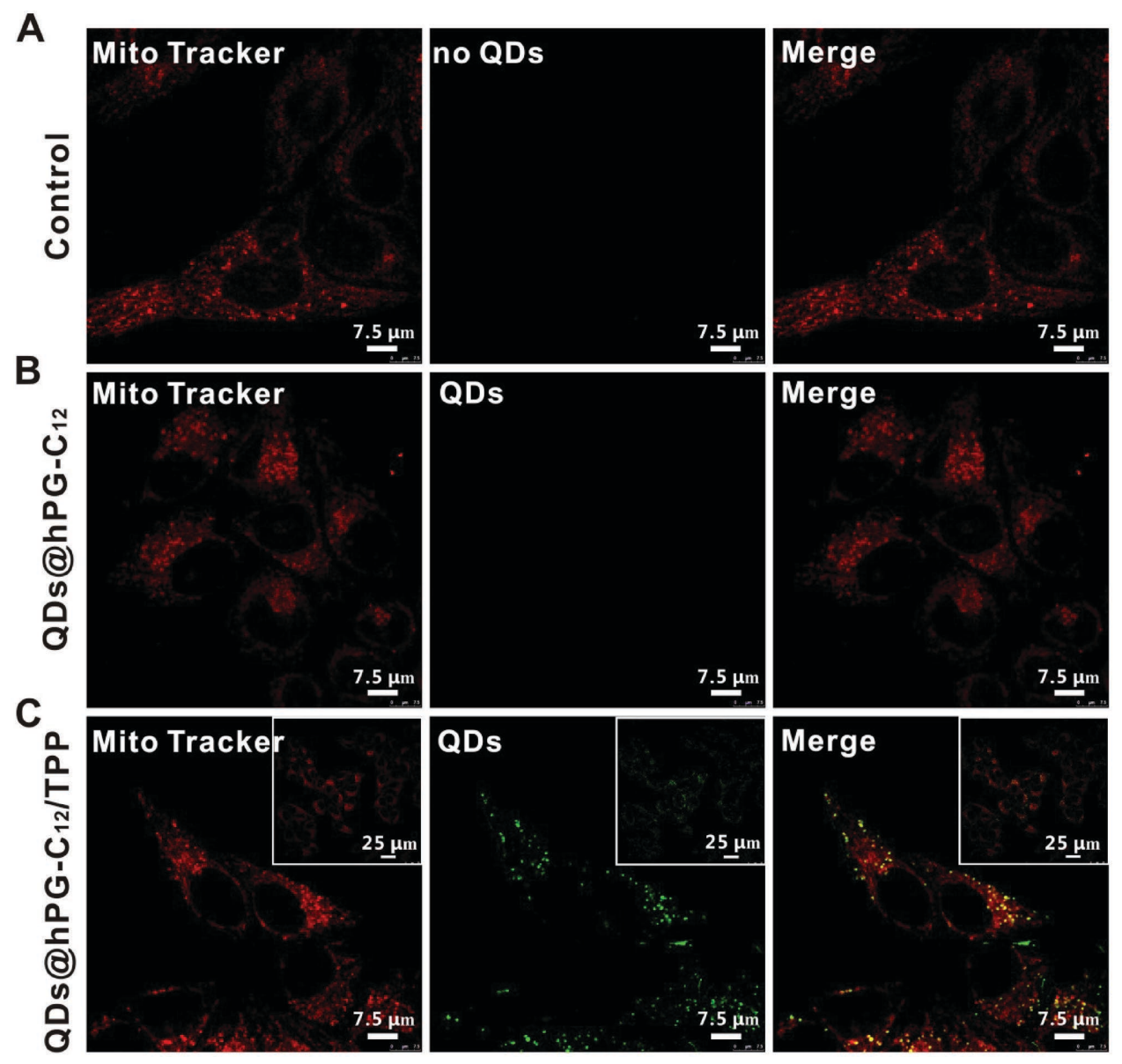

Figure 6. Subcellular localization of QDs@hPG-C $12 / T P P$ nanoprobes by confocal fluorescent images. A) Untreated HeLa cells stained with Mito Tracker Deep Red FM. B) HeLa cells treated with QDs@hPG-C 12 and Mito Tracker. C) HeLa cells treated with QDs@hPG-C $12 / T P P$ and Mito Tracker. The insets show broader field of view. Mito Tracker and QDs were set as red and green, respectively. Excitation wavelength: $453 \mathrm{~nm}$ for QDs and $633 \mathrm{~nm}$ for Mito Tracker.

from which we could further confirm that our constructed QDs@hPG-C 12 /TPP nanoprobes possessed excellent photostability and could serve as an alternative to currently commercially available mitochondria dyes. More importantly, the bright near-infrared fluorescence emission without photobleaching and mitochondria targeting ability make it a very promising nanoprobe for long-term and sensitive mitochondria imaging in living cells and mitochondria-related tumor diagnosis in vivo.

\subsection{Application of Drug-Loaded QDs@hPG-C $\mathrm{C}_{12} /$ TPP for Enhanced Chemotherapy In Vitro}

Compared with previously reported nanoprobe with single mitochondria imaging performance, our constructed flexible QDs@ hPG-C 12$_{2} / \mathrm{TPP}$ could be expanded as a versatile nanoplatform for therapeutic applications by further loading drugs, making it a promising cancer theranostic agent. Owing to the higher susceptibility of mitochondria in cancer cells to mitochondriaacting drugs, mitochondria have recently become interesting targets for tumor therapy. Targeted delivery of drugs to intracellular organelles of interest could be highly effective in maximizing the drug effects and enhancing treatment efficacy. ${ }^{[18]}$ To further prove the mitochondria-targeted ability and examine the versatility of this mitochondria-targeted system in cancer chemotherapy, anticancer drugs, doxorubicin (DOX) and betulinic acid (BA), were separately loaded to construct drug-loaded nanomedicine. The preparation of drug-loaded QDs@hPG-C 12 / TPP nanomedicine for precisely image-guided mitochondriaacted enhanced tumor chemotherapy is illustrated in Figure S12 (Supporting Information). The inhibitory effect of the drugloaded QDs@hPG-C 12 /TPP nanomedicine on the proliferation of HeLa cells was evaluated by the MTT assay. The pure drug and nontargeted drug-loaded nanomedicine were used as control. As shown in Figure S13 (Supporting Information), at the same drug concentration of 5 and $10 \mu \mathrm{g} \mathrm{mL} \mathrm{m}^{-1}$, mitochondria-targeted DOX-loaded QDs@hPG-C 12 /TPP nanomedicine exhibited obviously higher inhibited proliferation effect on HeLa cells than nontargeted DOX-loaded QDs@hPG-C 12 nanoprobes. The same phenomenon occurred when BA was loaded. All these mitochondria-targeted nanomedicine exhibited higher 
A

Time (s)

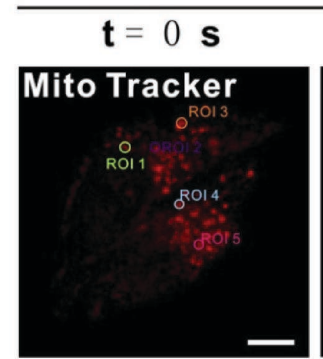

\section{$\mathbf{t}=178 \mathbf{s}$}

$\mathbf{t}=356 \mathbf{s}$

$\mathbf{t}=534 \mathbf{s}$
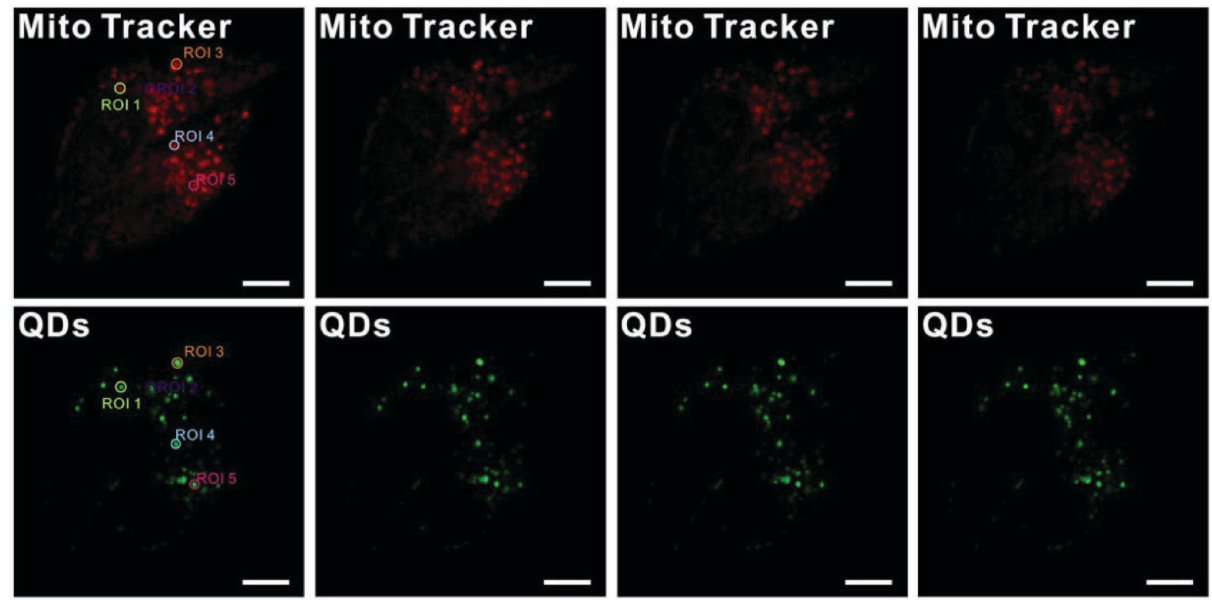

QDs
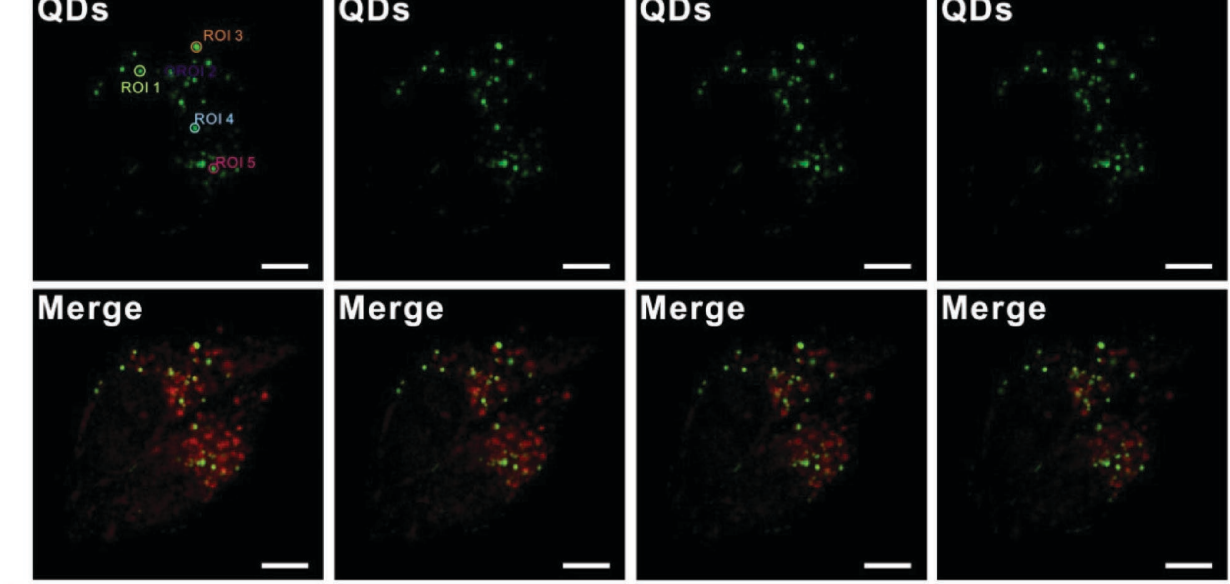

B

Mito Tracker
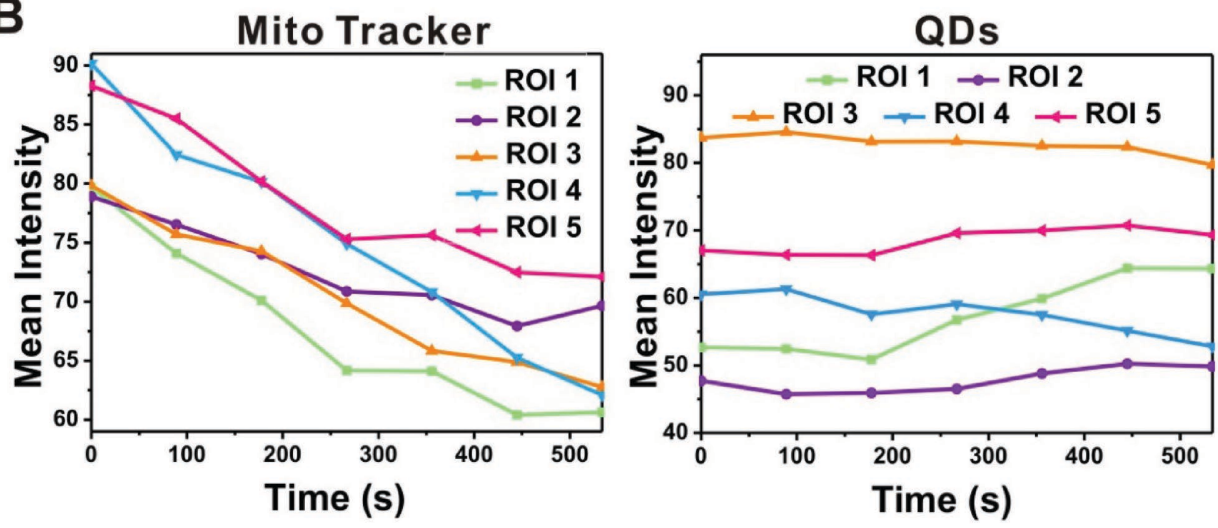

Figure 7. Photostability study of QDs@hPG-C 12 /TPP nanoprobe. A) Confocal fluorescent images of HeLa cells stained with QDs@hPG-C $12 / T P P$ and Mito Tracker Deep Red FM with increasing scanning time (0-8 min). Mito Tracker and QDs were set as red and green, respectively. Excitation wavelength: $453 \mathrm{~nm}$ (50\% laser power) for QDs and $633 \mathrm{~nm}$ (20\% laser power) for Mito Tracker Deep Red FM. Scale bar represents 7.5 m. B) The change of mean fluorescence intensity of Mito Tracker and QDs in the selected region of interest (ROI) in (A) over time.

anticancer activity than the nontargeted groups. Therefore, enhanced tumor cell killing efficacy was achieved with TPPfunctionalized QDs nanomedicine via specifically delivering drugs to mitochondria in our present work. The versatile drugloaded QDs nanoplatform could realize precisely image-guided mitochondria-acted enhanced chemotherapy efficacy. There was only a preliminary exploration in our present work, and detailed optimization of this nanoplatform should be further carried out. Moreover, it has been reported that mitochondrial-targeted drug therapy could help to overcome multidrug resistance in tumor chemotherapy, representing a promising approach to eradicate chemotherapy-refractory cancer cells. Therefore, our constructed versatile hyperbranched polyglycerol-based QDs nanoplatform has great potential for enhanced high-efficiency tumor theranostic. This nanoplatform could also be employed for targeted delivery of other mitochondria-acting drug such as lonidamine and $\alpha$-tocopheryl succinate to mitochondria of cells. Further explorations of its blood circulation stability, imaging ability, and anticancer effect in vivo need to be done before their translation to the clinic.

\section{Conclusions}

In conclusion, a highly photostable hyperbranched polyglycerol-based NIR QDs nanoplatform was successfully constructed 
and utilized for mitochondria-specific imaging and enhanced chemotherapy efficacy in HeLa cells. The QDs@hPG-C 12 / TPP nanoprobe was featured by uniform size, NIR fluorescence emission, good stability, low cytotoxicity, mitochondriatargeting ability, and superior photostability compared with commercial deep-red mitochondria dyes under continuous laser irradiation. Besides, drug-loaded QDs@hPG-C $12 / T P P$ displayed an obviously enhanced tumor cell killing efficacy than nontargeted ones. All these excellent advantages make this QDs@hPG-C 12 /TPP nanoplatform a promising candidate for long-term real-time mitochondria labeling and tracking. The near-infrared photostable fluorescence and cadmium and lead-free composition provide this nanoprobe more possibilities for clinical and translational applications. Moreover, it has great potential in the delivery of mitochondria-acting drugs for image-guided high-efficient treatment of tumor and other mitochondria-related diseases. We hope this work could open the door to the construction of multifunctional organelle-targeted theranostic nanoplatforms.

\section{Experimental Section}

Materials: hPG $\left(M_{\mathrm{n}}=11.1 \mathrm{~kg} \mathrm{~mol}^{-1}, M_{\mathrm{w}}=17.9 \mathrm{~kg} \mathrm{~mol}^{-1}, \mathrm{PDI}=1.61\right)$ was synthesized according to a previously published procedure. ${ }^{[16]}$ Sulfur (S, 99.9\%), indium(III) acetate $\left(\operatorname{In}(\mathrm{Ac})_{3}, 99.99 \%\right)$, copper(I) iodide (Cul, 99.999\%), zinc acetate dehydrate $\left(\mathrm{Zn}(\mathrm{Ac})_{2} \cdot 2 \mathrm{H}_{2} \mathrm{O}\right.$, reagent grade), oleic acid (OA, 90\%), oleylamine (97\%), DDT (98\%), 1-octadecene

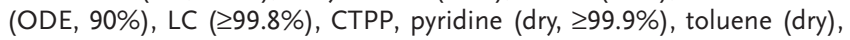
and dimethylformamide (DMF, dry) were purchased from Sigma-Aldrich (Germany). Methanesulfonyl chloride $(\mathrm{MsCl})$, triethylamine $\left(\mathrm{Et}_{3} \mathrm{~N}\right)$, sodium azide $\left(\mathrm{NaN}_{3}\right)$, triphenylphosphine, succinic anhydride (99.5\%), $\mathrm{NHS}, \mathrm{EDC} \cdot \mathrm{HCl}$, and MTT were procured from Acros (Germany) or Fluka (Germany). MitoTracker Deep Red FM was purchased from Invitrogen (German). All other reagents and solvents without statement were of reagent or HPLC grade and used without further treatment. For cell culture experiments, HeLa cells cultured in Dulbecco's Modified Eagle Medium (DMEM, supplemented with $1 \%$ penicillin streptomycin antibiotics, $10 \%$ fetal bovine serum, FBS) at $37{ }^{\circ} \mathrm{C}$ under a $5 \% \mathrm{CO}_{2}$ atmosphere were used. Ultrapure water (Millipore Inc., Bedford, MA) was used in all experiments and for preparation of all samples.

Preparation of ZCIS/ZnS QDs: Different batches of ZCIS/ZnS QDs with emission peak from 600 to $700 \mathrm{~nm}$ were prepared as previously described. ${ }^{[10 a, b]}$ In brief, $\mathrm{Zn}(\mathrm{Ac})_{2}(0.05 \mathrm{mmol})$, Cul ( $\left.0.1 \mathrm{mmol}\right), \ln (\mathrm{Ac})_{3}$ $(0.2 \mathrm{mmol})$, DDT $(1 \mathrm{~mL}), \mathrm{OA}(500 \mu \mathrm{L})$, and $\mathrm{ODE}(10 \mathrm{~mL})$ were mixed under stirring in a $50 \mathrm{~mL}$ round-bottom flask and heated to $120^{\circ} \mathrm{C}$ until a clear solution was formed. The reaction system was degassed for $20 \mathrm{~min}$, backfilled with $\mathrm{Ar}$, subsequently heated to $230^{\circ} \mathrm{C}$ followed by injection of $1.0 \mathrm{~mL}$ of sulfur precursor $(0.3 \mathrm{M}$ in ODE/oleylamine, the ratio is $2 / 1$ ), and finally kept at the temperature for $30 \mathrm{~min}$ for the growth of ZCIS/ZnS QDs core. In order to improve the fluorescence intensity of the QDs, $2 \mathrm{~mL}$ of $\mathrm{Zn}(\mathrm{Ac})_{2}$ stock solution ( $0.2 \mathrm{~m}$ in ODE/oleylamine, the ratio is $1 / 1$ ) was injected into the reaction mixture in four batches with a time interval of $15 \mathrm{~min}$ for the deposition of ZnS shell. The ZCIS/ZnS QDs were collected by adding acetone and centrifugation $(9000 \mathrm{rpm}$, $10 \mathrm{~min}$ ), purified two times again with chloroform/acetone mixture, and finally dispersed in chloroform and stored at $4{ }^{\circ} \mathrm{C}$ for further use.

Synthesis of Hyperbranched Polyglycerol- $C_{12}$ (hPG- $C_{12}$ ) and PolyglycerolhPG-TPP: The hyperbranched polyglycerol was prepared by a one-step ring-opening anionic polymerization as previously reported. ${ }^{20]}$

Synthesis of $h P G-C_{12}$ : The amphiphilic $\mathrm{hPG}-\mathrm{C}_{12}$ was prepared via an acyl chloride esterification method. Lauroyl chloride $(328 \mu \mathrm{L}, 1.42 \mathrm{mmol}$ ) dissolved in $6 \mathrm{~mL}$ of toluene was added dropwise to a solution of dried hyperbranched polyglycerol (1 $\mathrm{g}, 13.5 \mathrm{mmol}-\mathrm{OH}$ ) in $30 \mathrm{~mL}$ of pyridine. After stirring for $48 \mathrm{~h}$, most of the solvents were removed by rotary evaporation. The polymer was then subjected to dialysis against chloroform and methanol to remove toluene, pyridine, and free lauroyl chloride, and finally dried under vacuum to obtain dry products with a yield of $90 \%$. Determined by the ${ }^{1} \mathrm{HNMR}$, the grafting degree of $\mathrm{C}_{12}$ was about $10 \%$.

Synthesis of hPG-TPP: Due to the large steric hindrance of triphenylphosphonium groups, esterification of the terminal hydroxyl of hPG and the carboxyl of CTPP is very low. So amine functionalized hPG (hPG-amine) was first prepared here to increase the grafting degree of CTPP by a three-step protocol according to the previously reported method. ${ }^{[18]}$ In brief, the $\mathrm{OH}$ groups of polyglycerol was first conversed into mesyl (Ms) groups by reacting with methanesulfonyl chloride, conversed into azide $\left(\mathrm{N}_{3}\right)$ functionalities by reacting with $\mathrm{NaN}_{3}$, and finally reduced to primary amino $\left(\mathrm{NH}_{2}\right)$ groups by using triphenylphosphine as a reducing agent. hPG-amine with an amination degree of $10 \%$ was readily prepared and employed in our present work.

hPG-TPP was prepared on the basis of carbodiimide chemistry with $\mathrm{EDC} \cdot \mathrm{HCl}$ and $\mathrm{NHS}$ as the effective cross-linker. Typical procedure was conducted as follows: Briefly, hPG-amine $(300.0 \mathrm{mg}, 4.0 \mathrm{mmol}$ of primary hydroxyl and amine groups, 9:1) and CTPP (215.6 mg, $0.5 \mathrm{mmol}$ carboxyl groups) were dissolved in $15 \mathrm{~mL}$ of anhydrous DMF, followed by successively adding NHS (139.9 mg, $1.2 \mathrm{mmol})$ and EDC. $\mathrm{HCl}$ (232.5 mg, $1.2 \mathrm{mmol}$ ), which were 2.4 times the theoretical amount to ensure the conjugation efficiency. The mixture was kept at room temperature and protected from light under vigorous stirring for $24 \mathrm{~h}$, and then subjected to dialysis against methanol $(M W C O=2000)$ to remove the byproduct and free CTPP, and finally dried under vacuum to obtain dry products. Calculated from ${ }^{1} \mathrm{HNMR}$, the real grafting ratio of CTPP is about $5 \%$.

Determination of CAC: The amphiphilic $\mathrm{hPG}^{-\mathrm{C}_{12}}$ bears hydrophilic polyglycerol and hydrophobic alkyl chains. Herein, their self-assembly behavior, triggered by hydrophobic interactions among peripheral hydrophobic segment of alkyl chains, was studied by measuring CAC value with hydrophobic pyrene as a model fluorescence probe. The concentration of pyrene was set as $6.0 \times 10^{-7} \mathrm{M}$. The fluorescence emission spectra of pyrene when excited by an ultraviolet light at $338 \mathrm{~nm}$ were measured at various concentrations of polymer from 0 to $0.5 \mathrm{mg} \mathrm{mL}^{-1}$. The emission intensities at 373 and $384 \mathrm{~nm}$ were recorded.

Preparation of Mitochondria-Targeted QDs@hPG-C $12 / T P P$ and DrugLoaded QDs@hPG-C 12 /TPP: The QDs@hPG-C $12 / T P P$ nanoprobe was prepared via a previously reported ultrasonication-induced assembly method with minor modifications. ${ }^{[10 b]}$ In brief, $500 \mu \mathrm{L}$ of oil solution ( $6.0 \mathrm{mg} \mathrm{mL}^{-1}$ of QDs and $6.0 \mathrm{mg}$ of hPG-TPP in chloroform) was injected dropwise into $3 \mathrm{~mL}$ of cold hPG- $\mathrm{C}_{12}$ solution $\left(5.0 \mathrm{mg} \mathrm{mL}^{-1}\right.$ in water) under pulsed ultrasonication every $7 \mathrm{~s}$ for a duration of $3 \mathrm{~s}$ at 100-200 W for $8 \mathrm{~min}$ to form an emulsion. The solution was then evaporated to remove the residual chloroform until it was completely clear. The clear QDs@hPG- $\mathrm{C}_{12}$ solution was further purified by centrifugation at $20000 \mathrm{~g}$ for $20 \mathrm{~min}$ and washed to remove residual polymer and finally stored at $4{ }^{\circ} \mathrm{C}$ away from light. Herein, an ultrasonic cell crushing instrument with an ultrasonic booster (JY92-IID, Ningbo Scientz Biotechnology Co., LTD) was used.

The preparation of mitochondria-targeted QDs@hPG- $\mathrm{C}_{12}$ and drugloaded QDs@hPG-C 12 $_{12}$ TPP was conducted in a similar way, except that no hPG-TPP or extra drugs were added in the chloroform.

Size and Fluorescence Stability of QDs@hPG-C $12 / T P P$ : The size and fluorescence stability of nanoprobes in aqueous media are both very critical for their application for long-lime real imaging. In this work, to investigate whether the surface modification with hPG-based derivatives could provide a good stability, the size and fluorescence changes against time were monitored. The prepared mitochondria-targeted QDs@hPG$\mathrm{C}_{12} /$ TPP nanoprobe solution was gently mixed ultrapure $\mathrm{H}_{2} \mathrm{O}$, PBS buffer $(0.1 \mathrm{M}, \mathrm{pH}$ 7.4), and PBS buffer supplemented with 10\% FCS (PBS, $10 \%$ FCS), respectively. After different periods of incubation time, their average size was monitored by DLS using a Malvern Zetasizer. Every sample was measured at least three times and averaged. Besides, the fluorescent stability of QDs@hPG-C $12 / T P P$ nanoprobes was further 
investigated. The fabricated nanoprobe was incubated in PBS buffer $(0.01 \mathrm{M}, \mathrm{pH}=7.4)$ supplemented with $10 \% \mathrm{FCS}$, and its fluorescence emission intensity at different incubation time was measured by fluorescence spectrophotometer.

\section{Cytotoxicity, Cell Uptake, and Intracellular Studies}

Cell culture: Human cervical cancer HeLa cell line was used for cytotoxicity, cellular uptake, and intracellular studies as a model. The untreated cells served as negative control. The HeLa cell line was routinely cultured in DMEM (supplemented with $10 \%$ fetal bovine serum and $1 \%$ penicillin streptomycin antibiotics), incubated at $37^{\circ} \mathrm{C}$ in a humidified atmosphere with $5 \% \mathrm{CO}_{2}$, and split 1:4 three times a week.

Cytotoxicity assays: Cytotoxicity of the amphiphilic polymer hPG-C QDs@hPG-C 12 , and QDs@hPG-C $12 /$ TPP nanoprobes was evaluated by using the MTT assay. HeLa cells were seeded in a 96-well plate at a density of $5 \times 10^{3}$ cells per well in Dulbecco's Modified Eagle Medium and incubated at $37{ }^{\circ} \mathrm{C}$ in a humidified atmosphere with $5 \% \mathrm{CO}_{2}$ for $24 \mathrm{~h}$. The prepared samples were diluted in DMEM at serial concentrations of $12.5,25,50,100,200$, and $400 \mu \mathrm{g} \mathrm{mL} \mathrm{L}^{-1}$ and then added to replace the original culture medium. After a further incubation for $24 \mathrm{~h}$, $20 \mu \mathrm{L}$ of MTT stock solution $\left(5 \mathrm{mg} \mathrm{mL}^{-1}\right.$ in $\mathrm{H}_{2} \mathrm{O}$ ) was added per well for an additional $4 \mathrm{~h}$, and then replaced by $100 \mu \mathrm{L}$ of DMSO each well for $10 \mathrm{~min}$ under shaking. The UV absorbance of MTT at $570 \mathrm{~nm}$ was measured by a microplate reader. Cell viability was calculated by comparing the absorbance of cells treated with samples to that of nontreated cells.

Cell uptake of QDs@hPG-C $C_{12} / T P P$ nanoprobes measured by fluorescence microscopy and flow cytometry: The cell uptake and fluorescence imaging of the mitochondria-targeted QDs@hPG. $\mathrm{C}_{12} /$ TPP nanoprobes and nontargeted QDs@hPG- $\mathrm{C}_{12}$ nanoprobes were observed by fluorescence microscope with HeLa cells as a model. HeLa cells were seeded into a 24 -well plate at a density of $5.0 \times 10^{4}$ to $1.0 \times 10^{5}$ cells per well and incubated in DMEM medium supplemented with $1 \%$ penicillin/streptomycin and $10 \%$ FBS under a $5 \% \mathrm{CO}_{2}$ atmosphere at $37^{\circ} \mathrm{C}$. After incubation for $24 \mathrm{~h}$, about $30 \mu \mathrm{L}$ of QDs@hPG-C 12 and QDs@hPG-C $12 / T P P$ nanoprobe solutions were, respectively, added to the culture medium. After further incubation for predetermined time, the medium was removed and the cells were washed three times with PBS buffer. For cellular fluorescence imaging study, the cells were fixed with $4 \%$ paraformaldehyde for $20 \mathrm{~min}$ and then stained with $200 \mu \mathrm{L}$ of DAPI solution $\left(1 \mu \mathrm{g} \mathrm{mL} \mathrm{m}^{-1}\right)$ for 20 min for nucleus staining. Finally, the cells were washed twice with PBS buffer and subjected to fluorescence microscopy. In addition, flow cytometry was further used to quantify the cellular uptake of the QDs@hPG. $\mathrm{C}_{12}$ and QDs@hPG-C $\mathrm{C}_{12} /$ TPP nanoprobes with a flow cytometer on a FACSCalibur (BD Biosciences).

Intracellular mitochondria-specific imaging measured by confocal laser scanning microscopy: HeLa cells were seeded into a 24-well plate with glass slides on bottom for $24 \mathrm{~h}$. Next, nontargeted QDs@hPG- $\mathrm{C}_{12}$ and mitochondria-targeted QDs@hPG-C $12 / T P P$ nanoprobe solutions (100-200 $\mathrm{g} \mathrm{m} \mathrm{mL}^{-1}$ ) were, respectively, added to the culture medium for $1.5 \mathrm{~h}$ for the attachment of nanoprobes with the cell membrane. Then, the culture medium was replaced by fresh media and incubated for another $6 \mathrm{~h}$ for particle intracellular localization. For the co-localization study, the cells were further stained with MitoTracker Deep Red FM $\left(50 \times 10^{-9} \mathrm{M}\right)$ at $37^{\circ} \mathrm{C}$ for $30 \mathrm{~min}$, washed three times with PBS buffer, and finally imaged under Leica TCS SP5 CLSM.

Photostability of QDs@hPG-C 12 /TPP: To investigate the photostability of the QDs@hPG-C $12 / T P P$ nanoprobe, HeLa cells treated with QDs@ hPG-C $12 /$ TPP nanoprobe and MitoTracker were imaged by Leica TCS SP5 Confocal Microscope. QDs@hPG-C $C_{12} /$ TPP nanoprobes were excited at $453 \mathrm{~nm}$ (50\% laser power) and the fluorescence emission was collected from 550 to $700 \mathrm{~nm}$. The MitoTracker was excited at $633 \mathrm{~nm}$ (15\% laser power) and fluorescence emission was collected from 650 to $750 \mathrm{~nm}$.

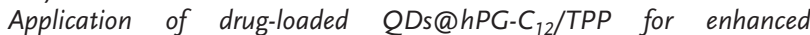
chemotherapy in vitro: Two anticancer drugs, DOX and BA, were separately loaded inside nontargeted QDs@hPG- $\mathrm{C}_{12}$ and mitochondria- targeted QDs@hPG-C 12 /TPP nanoprobes to construct drug-loaded nanomedicine to evaluate the therapeutic efficiency. The MTT assay was used to assess the cell viability. The cells were treated with 5 and $10 \mu \mathrm{g} \mathrm{mL}-1$ of pure drug and the nanomedicine at equivalent drug concentration at $37^{\circ} \mathrm{C}$ for $24 \mathrm{~h}$.

\section{Supporting Information}

Supporting Information is available from the Wiley Online Library or from the author.

\section{Acknowledgements}

C.D. and Z.L. contributed equally to this work. The authors gratefully acknowledge the National Natural Science Foundation of China (Grant Nos. 51373117, 51573128, and 51303126), the Key Project of Tianjin Natural Science Foundation (Grant Nos. 13JCZDJC33200 and 15JCQNJC03100), the National High Technology Program of China (Grant No. 2012AA022603), the Doctoral Base Foundation of the Educational Ministry of China (Grant No. 20120032110027), and SFB 1112 for financial support.

Received: February 26, 2016 Revised: March 29, 2016 Published online:

[1] S. Fulda, L. Galluzzi, G. Kroemer, Nat. Rev. Drug Discovery 2010, 9, 447.

[2] L. Rajendran, H.-J. Knölker, K. Simons, Nat. Rev. Drug Discovery 2010, 9, 29 .

[3] a) J. A. Canter, A. R. Kallianpur, F. F. Parl, R. C. Millikan, Cancer Res. 2005, 65, 8028; b) J. A. Petros, A. K. Baumann, E. Ruiz-Pesini, M. B. Amin, C. Q. Sun, J. Hall, S. Lim, M. M. Issa, W. D. Flanders, S. H. Hosseini, Proc. Natl. Acad. Sci. USA 2005, 102, 719.

[4] V. Gogvadze, S. Orrenius, B. Zhivotovsky, Trends Cell Biol. 2008, 18, 165

[5] a) S. Marrache, R. K. Pathak, S. Dhar, Proc. Natl. Acad. Sci. USA 2014, 111, 10444; b) G. R. Chamberlain, D. V. Tulumello, S. O. Kelley, ACS Chem. Biol. 2013, 8, 1389; c) J. L. Hickey, R. A. Ruhayel, P. J. Barnard, M. V. Baker, S. J. Berners-Price, A. Filipovska, J. Am. Chem. Soc. 2008, 130, 12570; d) R. K. Pathak, S. Marrache, D. A. Harn, S. Dhar, ACS Chem. Biol. 2014, 9, 1178

[6] A. Mallick, P. More, S. Ghosh, R. Chippalkatti, B. A. Chopade, M. Lahiri, S. Basu, ACS Appl. Mater. Interfaces 2015, 7, 7584.

[7] a) Q. Zhuang, H. Jia, L. Du, Y. Li, Z. Chen, S. Huang, Y. Liu, Biosens. Bioelectron. 2014, 55, 76; b) L. Wang, Y. Liu, W. Li, X. Jiang, Y. Ji, X. Wu, L. Xu, Y. Qiu, K. Zhao, T. Wei, Nano Lett. 2010, 11, 772.

[8] a) A. Chakraborty, N. R. Jana, J. Phys. Chem. C 2015, 119, 2888; b) A. M. Derfus, W. C. Chan, S. N. Bhatia, Adv. Mater. 2004, 16, 961

[9] a) F. Zhou, S. Wu, Y. Yuan, W. R. Chen, D. Xing, Small 2012, 8, 1543; b) F. Zhou, S. Wu, B. Wu, W. R. Chen, D. Xing, Small 2011, 7, 2727

[10] a) W. Guo, Theranostics 2013, 3, 99; b) C. Dong, Z. Liu, L. Zhang, W. Guo, X. Li, J. Liu, H. Wang, J. Chang, ACS Appl. Mater. Interfaces 2015, 7, 7566; c) Z. Liu, N. Chen, C. Dong, W. Li, W. Guo, H. Wang, S. Wang, J. Tan, Y. Tu, J. Chang, ACS Appl. Mater. Interfaces 2015, 7, 18997; d) W. Guo, X. Sun, O. Jacobson, X. Yan, K. Min, A. Srivatsan, G. Niu, D. O. Kiesewetter, J. Chang, X. Chen, ACS Nano 2015, 9, 488 . 
[11] X. He, J. Gao, S. S. Gambhir, Z. Cheng, Trends Mol. Med. 2010, 16, 574.

[12] a) Q. Xu, L. M. Ensign, N. J. Boylan, A. Schön, X. Gong, J.-C. Yang, N. W. Lamb, S. Cai, T. Yu, E. Freire, ACS Nano 2015, 9, 9217; b) C. D. Walkey, J. B. Olsen, H. Guo, A. Emili, W. C. Chan, J. Am. Chem. Soc. 2012, 134, 2139; c) R. Duncan, Nat. Rev. Cancer 2006, 6, 688; d) Y. Li, R. Lin, L. Wang, J. Huang, H. Wu, G. Cheng, Z. Zhou, T. MacDonald, L. Yang, H. Mao, J. Mater. Chem. B 2015, 3, 3591 .

[13] a) M. Calderón, M. A. Quadir, S. K. Sharma, R. Haag, Adv. Mater. 2010, 22, 190; b) Q. Wei, T. Becherer, S. AngiolettiUberti, J. Dzubiella, C. Wischke, A. T. Neffe, A. Lendlein, M. Ballauff, R. Haag, Angew. Chem. Int. Ed. 2014, 53, 8004; c) Y. Deng, J. K. Saucier-Sawyer, C. J. Hoimes, J. Zhang, Y.-E. Seo, J. W. Andrejecsk, W. M. Saltzman, Biomaterials 2014, 35, 6595; d) S. Reimann, D. Gröger, C. Kühne, S. B. Riese, J. Dernedde, R. Haag, Adv. Healthcare Mater. 2015, 4, 2154; e) Q. Qu, X. Ma, Y. Zhao, Nanoscale 2015, 7, 16677.

[14] a) A. P. Schenning, C. Elissen-Roman, J.-W. Weener, M. W. Baars, S. J. van der Gaast, E. Meijer, J. Am. Chem. Soc. 1998, 120, 8199; b) W.-I. Hung, C.-H. Chang, Y.-H. Chang, P.-S. Wu, C.-B. Hung,
K.-C. Chang, M.-C. Lai, S.-C. Hsu, Y. Wei, X.-R. Jia, Langmuir 2013, 29, 12075; c) B. N. Thota, L. H. Urner, R. Haag, Chem. Rev. 2015, 116, 2079.

[15] a) S. Marrache, S. Dhar, Proc. Natl. Acad. Sci. USA 2012, 109, 16288; b) C. W. T. Leung, Y. Hong, S. Chen, E. Zhao, J. W. Y. Lam, B. Z. Tang, J. Am. Chem. Soc. 2012, 135, 62; c) S. Marrache, S. Dhar, Chem. Sci. 2015, 6, 1832; d) H. S. Jung, J. Han, J.-H. Lee, J. H. Lee, J.-M. Choi, H.-S. Kweon, J. H. Han, J.-H. Kim, K. M. Byun, J. H. Jung, J. Am. Chem. Soc. 2015, 137, 3017.

[16] X. Wang, N. Shao, Q. Zhang, Y. Cheng, J. Mater. Chem. B 2014, 2, 2546.

[17] S. Roller, H. Zhou, R. Haag, Mol. Diversity 2005, 9, 305.

[18] a) S. Biswas, N. S. Dodwadkar, A. Piroyan, V. P. Torchilin, Biomaterials 2012, 33, 4773; b) R. Mo, Q. Sun, J. Xue, N. Li, W. Li, C. Zhang, Q. Ping, Adv. Mater. 2012, 24, 3659; c) M. Kodiha, Y. M. Wang, E. Hutter, D. Maysinger, U. Stochaj, Theranostics 2015, 5, 357.

[19] F. Paulus, M. E. Weiss, D. Steinhilber, A. N. Nikitin, C. Schütte, R. Haag, Macromolecules 2013, 46, 8458.

[20] D. Gröger, F. Paulus, K. Licha, P. Welker, M. Weinhart, C. Holzhausen, L. Mundhenk, A. D. Gruber, U. Abram, R. Haag, Bioconjugate Chem. 2013, 24, 1507. 